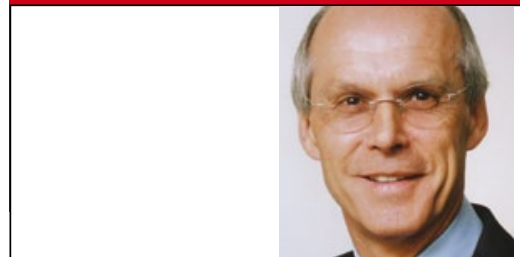

Prof. Dr. med. H. S. FüeßI Isar-AmperKlinikum, KI. München-Ost, Haar

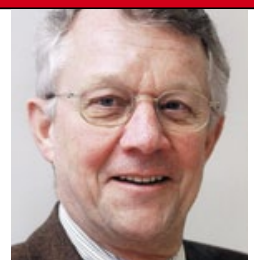

Prof. Dr. med. H. Holzgreve

Internist, Kardiologische Praxis, München

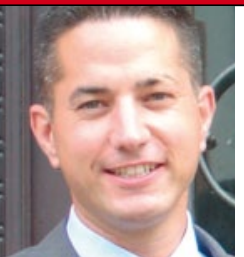

Prof. Dr. med. N. Diehm Inselspital Universität Bern

\title{
Aortenaneurysmen: weniger Verlaufskontrollen erforderlich
}

\section{Im Gegensatz zu den bisherigen Empfehlungen reichen bei kleinen Aortenaneurysmen Kontrollen im Abstand von mehreren Jahren aus.}

- Die Autoren sammelten die Verlaufsdaten von 15471 Patienten mit kleinen abdominalen Aortenaneurysmen mit Durchmessern von 3,0 bis 5,4 cm. Dazu wurden die Literatur gesichtet, auch Abstracts einbezogen und alle Autoren mit der Bitte um zusätzliche Informationen kontaktiert. Überwiegend diente die Sonografie zur Verlaufskontrolle, seltener die Computertomografie.

Die Größenzunahme der Aneurysmen war vom Ausgangswert abhängig: Bei einem Durchmesser von $3 \mathrm{~cm}$ betrug sie $1,28 \mathrm{~mm}$, bei $5 \mathrm{~cm}$ aber $3,61 \mathrm{~mm}$ pro Jahr. Bei anfänglichen Durchmessern von 3,4 und $5 \mathrm{~cm}$ besteht eine 10\%ige Chance auf Erreichen der kritischen Größe von 5,5 cm nach 7,4, 3,2 und 0,7 Jahren. Es wurden 178 bzw. 50 Rupturen bei 11262 Männern bzw. 1314 Frauen beobachtet. Aus dieser geringen Fallzahl errechnet sich für die Wahrscheinlichkeit einer Ruptur von $1 \%$ eine Dauer von mindestens zwei Jahren für Ausgangsdurchmesser von 3,0 bis 4,5 $\mathrm{cm}$ und von etwa 1,4 Jahren für Aus- gangswerte von $5,0 \mathrm{~cm}$. Bei gleichen Wachstumsraten für Männer und Frauen hatten Frauen ein vierfach höheres Rupturrisiko.

\section{- The RESCAN Collaboration}

Surveillance intervals for small abdominal aortic aneurysms. JAMA 309 (2013) 806813

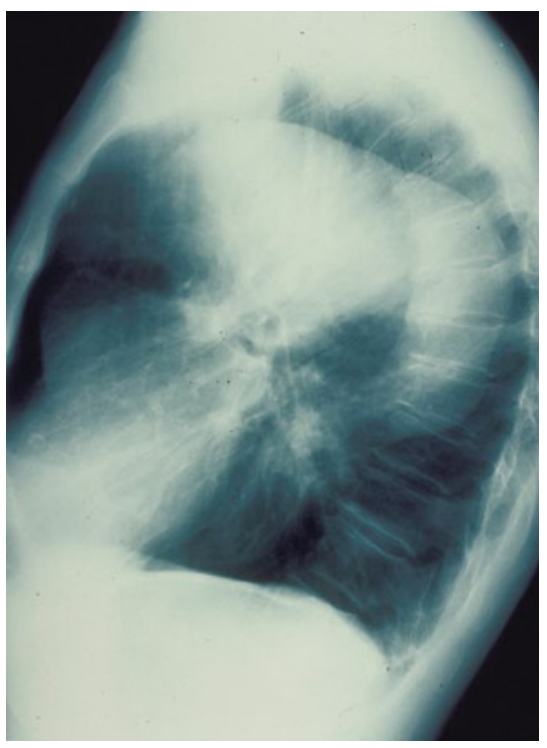

Aortenaneurysma im seitlichen RöntgenThorax.

\section{Kommentar}

Die Empfehlungen für Verlaufskontrollen bei Aortenaneurysmen sind sehr unterschiedlich. So werden bei Aneurysmen aller Größen zwischen 3,0 und 5,5 cm Abstände von zwölf Monaten genannt. Bei differenzierten Angaben werden für Durchmesser von 3,0-4,5 $\mathrm{cm} 12$ bis 24 Monate und über $4,5 \mathrm{~cm}$ drei bis sechs Monate vorgeschlagen. Die Autoren versuchen nun anhand einer Metaanalyse von 18 Studien mit über 15000 Patienten, objektive Daten für Empfehlungen zu Verlaufskontrollen zu geben. Das Resultat: Verlaufsuntersuchungen können bei Durchmessern von 3,0 bis $3,9 \mathrm{~cm}$ auf drei Jahre, von 4,0 bis $4,4 \mathrm{~cm}$ auf zwei Jahre und 4,5 bis 5,4 $\mathrm{cm}$ auf ein Jahr ausgedehnt werden. Damit kann das Rupturrisiko auf unter $1 \%$ gehalten werden. Mit diesem Vorgehen würde die Zahl der Kontrollen bei Aneurysmen über 3 $\mathrm{cm}$ von ca. 15 auf sieben reduziert. Die Alternative, die vorsorglich die Heterogenität der ausgewerteten Studien berücksichtigt, lautet: bei 3,0 bis 3,9cm alle zwei Jahre, bei 4,0 bis 4,9cm jährlich und bei 5,0 bis $5,4 \mathrm{~cm}$ alle sechs Monate. Dadurch würde die Zahl der Kontrollen bei Patienten mit Durchmessern ab $3 \mathrm{~cm}$ gegenüber den derzeitigen Empfehlungen immer noch um ein Drittel verringert.

H. HOLZGREVE = 